\title{
Sistema de reprodução em população natural de Chorisia speciosa A. St.-Hil. (Bombacaceae)
}

\author{
LINA M.F.I. SOUZA ${ }^{1}$, PAULO Y. KAGEYAMA² e ALEXANDRE M. SEBBENN ${ }^{3}$
}

(recebido: 22 de agosto de 2001; aceito: 8 de janeiro de 2003)

\begin{abstract}
Mating system in Chorisia speciosa A. St.-Hil. (Bombacaceae) natural population). This study aims to analyse by isozymes the outcrossing rate in a Chorisia speciosa population. Thus, open-pollinated seeds from 28 trees and leaves from 53 adult trees were sampled in a C. speciosa population at the Bauru Ecological Station, São Paulo State, Brazil. Eighteen alleles were observed distributed in seven polymorphic loci. The fixation index $(\hat{f})$ was smaller in adult trees than in families, suggesting selection against homozygous. The multilocus outcrossing estimate $\left(\hat{t}_{m}\right)$ was 0.816 , revealed that this species is a mixed mating system, predominantly alogamous. Heterogeneity of pollen pool was observed in outcrossing individual tree indicating that mating were not random. The paternity correlation $\left(\hat{r}_{p}\right)$ revealed that families are constituted of a high proportion of full sibs (71.4\%). There was also detection of crossing among relatives in population (5.6\%), indicating the possibility of family structure within population.
\end{abstract}

Key words - Chorisia speciosa, correlated matings, isozymes electrophoresis, outcrossing rate, population genetics

RESUMO - (Sistema de reprodução em população natural de Chorisia speciosa A. St.-Hil. (Bombacaceae)). O objetivo do presente trabalho foi avaliar a taxa de cruzamento de uma população de Chorisia speciosa por marcadores isoenzimáticos. Foram amostradas sementes de polinização aberta em 28 árvores e tecidos foliares em 53 árvores adultas de uma população de C. speciosa localizada na Estação Ecológica de Bauru, São Paulo, Brasil. Observou-se 18 alelos distribuídos em sete locos polimórficos. O índice de fixação $(\hat{f})$ foi menor nos adultos em relação às progênies, sugerindo seleção contra homozigotos. A estimativa da taxa de cruzamento multilocos $\left(\hat{t}_{m}\right)$ foi de 0,816 , revelando que a espécie é de reprodução mista, predominantemente alógama. Foi detectada heterogeneidade no conjunto de pólen dos cruzamentos, indicando que os mesmos não foram aleatórios. Em concordância, a correlação de paternidade $\left(\hat{r}_{p}\right)$ revelou que as progênies são constituídas por uma alta proporção de irmãos completos $(71,4 \%)$. Foram também detectados cruzamentos entre aparentados na população $(5,6 \%)$, sugerindo uma possível estruturação espacial em famílias.

Palavras-chave - Chorisia speciosa, eletroforese de isosimas, genética de populações, linhagens correlacionadas, taxa de crescimento

\section{Introdução}

O sistema de reprodução é a forma como uma espécie deixa seus descendentes. A reprodução pode ocorrer por cruzamentos aleatórios, cruzamentos biparentais, autofecundações, apomixia e suas combinações. O sistema de reprodução, juntamente com os mecanismos de dispersão de pólen e sementes, tem papel central na determinação da estrutura genética de populações (Hamrick \& Lovelless 1986). Estudos do sistema de reprodução em espécies arbóreas tropicais, por marcadores isoenzimáticos, têm mostrado que a grande maioria é alógama ou de sistema misto, com predomínio de alogamia (Bawa \& O’Malley 1985,

\footnotetext{
1. R. Carlos de Campos, 282, 13416-395 Piracicaba, SP, Brasil.

2. USP/ESALQ, Av. Pádua Dias, 11, 13418-900 Piracicaba, SP, Brasil.

3. Instituto Florestal, Caixa Postal 1322, 01059-970 São Paulo, SP, Brasil.
}

Murawski et al. 1990, Murawski 1995). Espécies de cruzamento mantêm a maior parte de sua variabilidade genética distribuída dentro de populações, diferente das espécies predominantemente de autofecundação, onde a maior parte está entre populações (Hamrick 1983, Hamrick \& Godt 1989). Portanto, a forma de reprodução determina em parte a estrutura genética espacial e temporal das populações (Hamrick 1983). Seu conhecimento é de fundamental importância em programas de melhoramento e conservação genética porque permite delinear estratégias que otimizem a amostragem da variabilidade genética e a adoção de modelos genéticos-estatísticos adequados para a estimativa de parâmetros genéticos.

Chorisia speciosa A. St.-Hil. (Bombacaceae) é uma espécie arbórea tropical de grande porte e ampla distribuição geográfica. A espécie comumente apresenta $10 \mathrm{~m}$ a $15 \mathrm{~m}$ de altura e $30 \mathrm{~cm}$ a $60 \mathrm{~cm}$ de diâmetro à altura do peito (DAP - 1,3 m), podendo atingir $20 \mathrm{~m}$ a $30 \mathrm{~m}$ de altura e $120 \mathrm{~cm}$ de DAP. Sua área de ocorrência 
abrange principalmente as Florestas Mesófilas Semidecíduas, entre as latitudes $12^{\circ} \mathrm{S}$ a $30^{\circ} \mathrm{S}$, na Argentina, Paraguai e Brasil. C. speciosa também é cultivada em regiões tropicais e subtropicais, no hemisfério norte, até as Antilhas e o sul dos Estados Unidos. As flores são hermafroditas e o fruto é uma cápsula oblonga deiscente. As sementes são envoltas por pêlos (paina), que auxiliam a dispersão pelo vento. Cada árvore produz em média 300 a 700 frutos cada um com cerca de 120 a 200 sementes (Carvalho 1994). Os prováveis polinizadores são borboletas (Castillo 1986), morcegos e/ou beija-flores (Morellato 1991). As sementes são dispersas pelo vento (anemocoria), atingindo distâncias superiores a $160 \mathrm{~m}$; porém, a maior densidade de sementes é observada nas vizinhanças da matriz (Castillo 1986).

O objetivo deste estudo foi investigar o sistema de reprodução de uma população natural de Chorisia speciosa a partir da análise de eletroforese de isoenzimas. Para tanto, coletaram-se sementes de polinização livre em 28 árvores e tecidos foliares em 53 árvores adultas reprodutivas na Estação Ecológica de Bauru, SP. A comparação da geração adulta e de progênies permitiu a realização de testes de hipóteses referentes ao sistema de reprodução da espécie, como teste de Equilíbrio de Hardy-Weinberg, homogeneidade entre as freqüências alélicas do pólen vs. óvulos, adultos vs. pólen, adultos vs. óvulos e adultos vs. progênies.

\section{Material e métodos}

O estudo foi realizado em um fragmento de Floresta Mesófila Semidecídua, de propriedade do Instituto Florestal, Estação Ecológica de Bauru, SP. A Estação ocupa uma área de 287,28 hectares e localiza-se nas coordenadas $22^{\circ} 19^{\prime} \mathrm{S}$ e $49^{\circ} 04^{\prime}$ 'W. Em levantamento prévio do número de exemplares reprodutivos de Chorisia speciosa, foram identificadas 92 árvores, revelando uma densidade populacional de aproximadamente um indivíduo reprodutivo a cada três hectares. Desses 92 indivíduos, para a caracterização do sistema de reprodução, foram coletadas sementes de polinização livre, aleatoriamente em 28 árvores (30\% da população reprodutiva). De cada árvore, foram coletadas sementes de vários frutos, as quais foram armazenadas em sacos plásticos, separadas e identificadas pela árvore materna e transportadas para o viveiro florestal do Departamento de Ciências Florestais da ESALQ/USP. Posteriormente, essas sementes foram germinadas em casa de vegetação. Para a análise de eletroforese, foram avaliadas 20 plântulas de cada progênie, com nove meses de idade. Também foram amostrados aleatoriamente 53 indivíduos adultos reprodutivos ( $58 \%$ da população reprodutiva), cujo diâmetro à altura do peito (DAP) variou de $35 \mathrm{~cm}$ a $134 \mathrm{~cm}$. A distribuição espacial dos indivíduos na população era agregada, formando grupos parcialmente isolados. Procurou-se representar esta distribuição, amostrando-se vários indivíduos dentro de cada agregado.

A eletroforese de isoenzimas, realizada no Laboratório de Reprodução e Genética de Espécies Arbóreas (Largea) do Departamento de Ciências Florestais da ESALQ/USP, em Piracicaba, SP, foi horizontal, conduzida em meio suporte de gel de amido de milho (penetrose 30 ) a $13 \%$. O protocolo de revelação das isoenzimas foi determinado com base em Kephart (1990) e Alfenas (1998). Inicialmente, foram testados vários tampões de extração, tampão de cuba e gel e sistemas isoenzimáticos de coloração. O tampão de extração número 1 de Alfenas (1998), modificado pela ausência de L-ácido ascórbico, bissulfito de sódio, borato de sódio e PEG-2000, foi o que se mostrou mais adequado para a espécie. O tampão de cuba e gel que apresentou o melhor padrão foi o Citrato Morfolina (CM, pH 6,1 - Clayton \& Tretiak 1972). Foram testados 15 sistemas isoenzimáticos de coloração, sendo selecionados os seis que apresentaram o melhor padrão de interpretação dos zimogramas e que suportavam uma segregação mendeliana codominante. Os locos aparentes foram definidos como regiões do zimograma que apresentavam um comportamento aparentemente independente dos demais e que ostentassem uma segregação mendeliana aparente. A coerência entre genitor feminino (receptor de pólen) e progênie proporciona a devida consistência a essa forma de interpretação. Os sistemas enzimáticos com revelação de mais de um loco, foram denominados alfa-numericamente, de forma crescente, dos locos mais catódicos para os mais anódicos. Para os alelos, denominou-se o mais freqüente com valor um (1), e de forma crescente, os alelos mais catódicos para os mais anódicos.

As enzimas foram extraídas macerando-se as folhas com nitrogênio líquido, acrescentando-se cinco a sete gotas da solução de extração número 1 de Alfenas (1998), modificada para este estudo, como já apresentado. Os seis sistemas isoenzimáticos revelados foram: Fosfogluco Isomerase (PGI-E.C. 5.3.1.9), Malato Desidrogenase (MDH-E.C. 1.1.1.37), Peroxidase (PRX-E.C. 1.11.1.7), Xiquimato Desidrogenase (SKDH-E.C. 1.1.1.25), Leucina Aminopeptidase (LAP - E.C.3.4.11.1) e Fosfoglucomutase (PGM - E.C.5.4.2.2.). As receitas de revelação das isoenzimas encontram-se em Alfenas (1998).

O sistema de reprodução da população de $C$. speciosa foi analisado com base nos modelos de cruzamentos mistos de Ritland \& Jain (1981) e cruzamentos correlacionados (Ritland, 1989), com o auxílio do programa "Multilocos MLTR" (Ritland 1997). Foram estimadas: 1) a taxa populacional de cruzamento multilocos $\left(\hat{t}_{m}\right)$, pelo método de máxima verossimilhança (Expectation-Maximization EM); 2) a taxa populacional de cruzamento unilocos $\left(\hat{t}_{s}\right)$; $3)$ a taxa de cruzamento entre aparentados $\left.\left(\hat{t}_{m}-\hat{t}_{s}\right) ; 4\right)$ a taxa individual de cruzamento multilocos $(\hat{t}), 5)$ as freqüências alélicas dos óvulos e do pólen ( $o$ e $p)$; 6) o índice de fixação 
nas árvores maternas $\left.\left(\hat{F}_{m}\right) ; 7\right)$ a correlação de autofecundação $\left(\hat{r}_{s}\right)$ e; 8$)$ a correlação de paternidade $\left(\hat{r}_{p}\right)$. As pressuposições do modelo misto são dados em Ritland \& Jain (1981). O erro padrão das estimativas dos parâmetros foi obtido por 1.000 reamostragens bootstraps, onde a unidade de amostragem para as estimativas das taxas de cruzamento individual e populacional foram as plantas dentro das progênies e as progênies, respectivamente. O teste de cruzamentos aleatórios foi avaliado pelo teste de Equilíbrio de Hardy-Weinberg, teste de homogeneidade entre as freqüências alélicas dos adultos vs. progênies, adultos vs. pólen, adultos vs. óvulos e óvulos vs. pólen, calculando-se o estimador $\hat{F}_{S T}$ (Nei 1977) apenas como uma medida de divergência genética entre freqüências alélicas de diferentes grupos. Para testar a significância de $\hat{F}_{S T}$, para cada loco, aplicou-se o teste de qui-quadrado $\left[\mathrm{c}^{2}=2 n \hat{F}_{S T}(k-1)\right.$; GL $=$ $(k-1)(s-1)]$, proposto por Workman \& Niswander (1970), em que: $n=$ número de indivíduos nos dois grupos, $k=$ número de alelos e $s=$ número de grupos. Os índices de fixação para adultos $(\hat{F})$ e progênies $\left(\hat{F}_{p}\right)$ e o coeficiente médio de coancestria dentro de progênies $\left(\hat{\theta}_{p}\right)$ foram obtidos pela análise da variância das freqüências gênicas da população e seus respectivos intervalos de confiança por 10.000 reamostragens bootstrap, estimativas obtidas pelo programa GDA (Lewis \& Zaykin 1999).

\section{Resultados}

Todos os locos avaliados foram polimórficos tanto nos adultos como nas progênies, segregando de dois a quatro alelos (tabela 1). Não foram detectados alelos exclusivos entre a geração adulta e a geração de progênies. Contudo, quatro alelos de baixa freqüência nos adultos (alelo 4 na Pgi-1, alelo 3 na Pgm-1 e Mdh-3 e alelo 1 na Lap-1) reduziram ainda mais suas freqüências nas progênies, sugerindo que durante o processo de reprodução possa ter ocorrido deriva genética. As estimativas das probabilidades do teste exato de Fisher (tabela 2) revelaram desvios das proporções do Equilíbrio de Hardy-Weinberg (EHW) em um loco nos adultos (Prx-2) e em três locos nas progênies (Skdh-1, Prx-1 e Prx-2), indicando desvios de cruzamentos aleatórios e/ou a atuação de forças evolucionárias. A estimativa da divergência genética $\left(\hat{F}_{S T}\right)$ entre as freqüências alélicas do pólen vs. óvulos foi significativa em seis dos sete locos avaliados (tabela 2), reforçando a hipótese de desvios de cruzamentos aleatórios na população. Por outro lado, a estimativa da divergência genética entre as freqüências alélicas dos adultos vs. progênies (tabela 2) foi baixa em todos os locos, mas significativas para os locos Pgi-1 e Prx-1, sugerindo pequena alteração nas freqüências
Tabela 1. Estimativa das freqüências alélicas, número total de alelos $\left(n_{a}\right)$ e tamanho amostral $(n)$ em árvores adultas, pólen, óvulos e progênies de C. speciosa.

Table 1. Estimation of allelic frequencies, total number of alleles $\left(n_{a}\right)$ and sample size $(n)$ in adult trees, pollen, ovules and progenies of $C$. speciosa.

\begin{tabular}{lccccc}
\hline Loco & Alelo & Adultos & Pólen & Óvulos & Progênies \\
\hline Skdh-1 & 1 & 0,845 & 0,810 & 0,948 & 0,872 \\
& 2 & 0,155 & 0,190 & 0,052 & 0,128 \\
Pgi-1 & 1 & 0,490 & 0,437 & 0,424 & 0,410 \\
& 2 & 0,281 & 0,310 & 0,390 & 0,364 \\
& 3 & 0,156 & 0,249 & 0,169 & 0,224 \\
& 4 & 0,073 & 0,003 & 0,017 & 0,002 \\
Pgm-1 & 1 & 0,837 & 0,736 & 0,862 & 0,830 \\
& 2 & 0,096 & 0,230 & 0,103 & 0,142 \\
& 3 & 0,067 & 0,034 & 0,034 & 0,028 \\
Mdh-3 & 1 & 0,872 & 0,774 & 0,881 & 0,862 \\
& 2 & 0,117 & 0,206 & 0,102 & 0,130 \\
& 3 & 0,011 & 0,020 & 0,017 & 0,008 \\
Prx-1 & 1 & 0,660 & 0,704 & 0,810 & 0,784 \\
& 2 & 0,340 & 0,296 & 0,190 & 0,216 \\
Prx-2 & 1 & 0,429 & 0,524 & 0,483 & 0,514 \\
& 2 & 0,571 & 0,476 & 0,517 & 0,486 \\
Lap-1 & 1 & 0,091 & 0,003 & 0,017 & 0,054 \\
& 2 & 0,909 & 0,997 & 0,983 & 0,946 \\
$n_{a}$ & & 18 & 18 & 18 & 18 \\
\hline & & 53 & - & 28 & 420 \\
\hline
\end{tabular}

alélicas entre as duas gerações, devido à atuação de forças evolucionários, provavelmente deriva genética. As estimativas das divergências genéticas entre as freqüências alélicas dos adultos vs. pólen e adultos vs. óvulos (tabela 2) foram baixas (máximo 4,3\%), mas significativas em quase todos os locos. Estes resultados indicam que as freqüências alélicas do pólen efetivamente cruzado e das matrizes que deram origem às progênies não eram perfeitamente representativas da população, em especial no caso do pólen.

Ambas gerações de adultos e progênies apresentaram estimativas dos índices de fixação positivos e altos (tabela 3 ), mas apenas o índice estimado para as progênies $\left(\hat{F}_{p}=0,259 \pm 0,220\right)$ foi estatisticamente diferente de zero. A ausência de endogamia nos adultos e excesso de homozigotos nas progênies sugere a presença de seleção contra homozigotos. A estimativa do coeficiente de coancestralidade $\left(\hat{\theta}_{F}\right)$ médio dentro de progênies 
Tabela 2. Estimativa das probabilidades exatas de Fisher $(P)$ para o Equilíbrio de Hardy-Weinberg em adultos e progênies, divergência genética $\left(\hat{F}_{S T}\right)$ entre freqüências alélicas dos adultos vs. pólen, adultos vs. óvulos e pólen vs. óvulos, em uma população de C. speciosa. Entre parênteses são dados os graus de liberdade.

Table 2. Estimation of Fischer's exact probabilities $(P)$ for Hardy-Weinberg equilibrium in adults and progenies, genetic divergence $\left(\hat{F}_{S T}\right)$ between allelic frequencies of adults vs. pollen, adults vs. ovules, and pollen vs. ovules, into a population of C. speciosa. Degrees of freedom are parenthesized.

\begin{tabular}{|c|c|c|c|c|c|c|c|}
\hline \multicolumn{4}{|c|}{ EHW } & \multicolumn{4}{|c|}{$\hat{F}_{S T}$} \\
\hline \multirow{2}{*}{$\begin{array}{l}\text { Loco } \\
\text { Skdh-1 }\end{array}$} & \multicolumn{2}{|c|}{ Adultos } & Progênies & $\begin{array}{l}\text { Adultos vs. } \\
\text { Progênies }\end{array}$ & $\begin{array}{l}\text { Adultos vs. } \\
\text { Pólen }\end{array}$ & $\begin{array}{l}\text { Adultos vs. } \\
\text { Óvulos }\end{array}$ & $\begin{array}{c}\text { Pólen vs. } \\
\text { Óvulos }\end{array}$ \\
\hline & 1,000 & (1) & $0,000^{* *}$ (1) & 0,001 & $0,002 \quad$ (1) & $0,029 * * \quad(1)$ & $0,179^{* *}$ \\
\hline Pgi-1 & 1,000 & (6) & $0,925 \quad$ (6) & $0,003^{*}$ & $0,007 * *$ & $0,008^{* *}$ & $0,042 * *$ \\
\hline Pgm-1 & 0,604 & (3) & 0,314 & $0,001 \quad$ (1) & $0,021^{* *}$ (1) & 0,002 & $0,108 * *$ \\
\hline Mdh-3 & 1,000 & (3) & 0,380 & $0,000 \quad$ (1) & $0,015^{* *}$ (1) & 0,000 & $0,082 * *$ \\
\hline Prx-1 & 0,205 & (1) & $0,000^{* *}(1)$ & $0,011^{*}$ & $0,002 \quad(1)$ & $0,029 * * \quad(1)$ & $0,020 * *$ \\
\hline Prx-2 & $0,004 * *$ & (1) & $0,000 * * \quad(1)$ & $0,003 \quad$ (1) & $0,009^{*} \quad(1)$ & $0,003^{*} \quad(1)$ & $0,06 * *$ \\
\hline Lap-1 & 0,220 & (1) & 1,000 & $0,003 \quad$ (1) & $0,043^{* *}$ (1) & $0,027^{* *} \quad(1)$ & 0,007 \\
\hline
\end{tabular}

$*: P \leq 0,05 . * *: P \leq 0,01$.

assumiu valores próximos aos esperados em progênies de irmãos completos $(0,253 \pm 0,146)$ (tabela 3$)$, corroborando com a hipótese de desvios de cruzamentos aleatórios.

As estimativas das taxas de cruzamentos individuais variaram de 0,12 a 1,0 , sendo que, de acordo com as estimativas dos intervalos de confiança a $95 \%$ de probabilidade, $35,7 \%$ dos valores foram significativamente menores do que 1,0 (tabela 3 ). A estimativa da taxa de cruzamento multilocos $\left(\hat{t}_{m}\right)$ foi estatisticamente diferente de $1,0(0,816 \pm 0,040)$, a julgar pelo erro padrão da média, revelando que C. speciosa é de reprodução mista, predominantemente alógama. A estimativa da taxa de cruzamento unilocos $\left(\hat{t}_{s}\right)$ foi alta e significativamente diferente da unidade $(0,76 \pm 0,047)$, mas inferior à estimativa da taxa de cruzamento multilocos, confirmando que a espécie é de sistema misto predominantemente de cruzamento e sugerindo a possibilidade de ter ocorrido cruzamentos entre indivíduos aparentados na população. A taxa de cruzamento estimada entre aparentados $\left(\hat{t}_{m}-\hat{t}_{s}\right)$ foi baixa $(5,6 \%)$, mas significativamente diferente de zero, considerando o erro padrão da média. A estimativa da correlação de autofecundação $\left(\hat{r}_{s}\right)$ foi baixa $(0,160 \pm 0,048)$, mas significativa, indicando que nessa população de $C$. speciosa não existe grande tendência de algumas plantas deixarem mais descendentes por autofecundação ou cruzamentos, sendo os indivíduos de autofecundação distribuídos aleatoriamente entre as progênies. A estimativa da correlação de paternidade
$\left(\hat{r}_{P}\right)$ foi alta e estatisticamente diferente de zero $(0,875 \pm 0,063)$, evidenciando que grande parte das progênies de cruzamentos foram geradas por cruzamentos biparentais, ou seja, foram geradas pelo mesmo parental materno e paterno.

\section{Discussão}

A estimativa da taxa de cruzamento multilocos mostrou que Chorisia speciosa A. St.-Hil. é de reprodução mista, com predominância de alogamia $\left(\hat{t}_{m}=0,816 \pm 0,040\right)$. A taxa de cruzamento individual estimada por árvore materna variou entre plantas, sendo inferior a 0,5 em nove das 28 progênies e significativamente diferentes de 1,0 em 10 progênies. A taxa de cruzamento em plantas hermafroditas, como C. speciosa, depende de fatores tais como: presença e intensidade de mecanismos de auto-incompatibilidade; grau de protogenia e protandria; comportamento forrageiro dos polinizadores entre e dentro de árvores, o qual é influenciado pela densidade de florescimento das árvores na população e; aborto seletivo de frutos e sementes de autofecundação (Murawski \& Hamrick 1991). Em C. speciosa existem indícios de auto-incompatibilidade. Gibbs \& Bianchi (1993), estudando o evento de pós-polinização de árvores cultivadas de $C$. speciosa, observaram a formação do tubo polínico e penetração do óvulo, tanto para flores de autopolinização quanto para aquelas de 
Tabela 3. Estimativa da taxa de cruzamento multilocos individual por planta materna $(\hat{t})$ e parâmetros do sistema de reprodução em uma população de C. speciosa. $(\hat{t})$ : Intervalo de confiança, a 95\% de probabilidade pelo teste t de Student. [ ]: Erro padrão da média, obtido por 1.000 reamostragens "bootstrap".

Table 3. Estimate of rates of individual multiloci crossings per maternal plant $(\hat{t})$ and parameters of the reproductive system in a population of $C$. speciosa. $(\hat{t})$ : 95\% confidence interval using Student's t test. [ ]: Mean standard error for 1,000 "bootstrap" repetitions.

\begin{tabular}{|c|c|c|c|c|c|}
\hline Progênies & $n$ & $\hat{t}$ & Progênies & $n$ & $\hat{t}$ \\
\hline 1 & 20 & $0,68(0,38)$ & 15 & 20 & $0,42(0,42)$ \\
\hline 2 & 20 & $1,00(0,00)$ & 16 & 20 & $0,89(0,24)$ \\
\hline 3 & 20 & $1,00(0,00)$ & 17 & 20 & $0,12(0,66)$ \\
\hline 4 & 20 & $0,22(0,50)$ & 18 & 17 & $0,94(0,36)$ \\
\hline 5 & 20 & $0,56(0,44)$ & 19 & 20 & $0,78(0,42)$ \\
\hline 6 & 20 & $0,90(0,34)$ & 20 & 20 & $0,43(0,46)$ \\
\hline 7 & 20 & $0,39(0,70)$ & 21 & 19 & $0,88(0,23)$ \\
\hline 8 & 19 & $0,99(0,14)$ & 22 & 20 & $1,00(0,00)$ \\
\hline 9 & 20 & $0,49(0,30)$ & 23 & 20 & $0,27(0,52)$ \\
\hline 10 & 19 & $0,88(0,14)$ & 24 & 20 & $0,74(0,36)$ \\
\hline 11 & 10 & $0,57(0,42)$ & 25 & 19 & $0,51(0,38)$ \\
\hline 12 & 11 & $0,78(0,36)$ & 26 & 20 & $0,59(0,62)$ \\
\hline 13 & 12 & $1,00(0,00)$ & 27 & 17 & $1,00(0,00)$ \\
\hline 14 & 10 & $0,21(0,34)$ & 28 & 20 & $0,35(0,34)$ \\
\hline \multicolumn{5}{|c|}{ Índice de fixação nas árvores maternas $\left(\hat{F}_{-}\right)$} & $0,073[0,051]$ \\
\hline \multicolumn{5}{|c|}{ Índice de fixação na árvores adultas $(\hat{F})$} & $0,142(0,211)$ \\
\hline \multicolumn{5}{|c|}{ Índice de fixação nas progênies $(\hat{F})$} & $0,259(0,220)$ \\
\hline \multicolumn{5}{|c|}{ Coeficiente de coancestralidade dentro de progênies $\left(\hat{\theta}_{F}\right)$} & $0,253(0,146)$ \\
\hline \multicolumn{5}{|c|}{ Taxa de cruzamento unilocos $\left(\hat{t}_{s}\right)$} & $0,760[0,047]$ \\
\hline \multicolumn{5}{|c|}{ Taxa de cruzamento multilocos $\left(\hat{t}_{m}\right)$} & $0,816[0,040]$ \\
\hline \multicolumn{5}{|c|}{ Taxa de cruzamento entre aparentados $\left(\hat{t}_{m}-\hat{t}_{s}\right)$} & $0,056[0,023]$ \\
\hline \multicolumn{5}{|c|}{ Correlação de autofecundação $\left(\hat{r}_{s}\right)$} & $0,160[0,048]$ \\
\hline \multicolumn{5}{|c|}{ Correlação de paternidade $\left(\hat{r}_{p}\right)$} & $0,875[0,063]$ \\
\hline
\end{tabular}

polinização cruzada, porém $100 \%$ das flores autopolinizadas foram abortadas posteriormente (auto-incompatibilidade tardia). A discordância nos resultados aqui obtidos, em relação aos obtidos por Gibbs \& Bianchi (1993) pode estar associada aos diferentes métodos utilizados para avaliar o sistema de reprodução da espécie, ou a presença de variabilidade genética para auto-incompatibilidade. A avaliação do sistema de reprodução, a partir de dados de marcadores codominantes (isoenzimas, microssatélites e RFLP), é robusta, quando um número mínimo de três a quatro locos, com freqüências alélicas intermediária $\left(1 . n_{a}^{-1}\right.$, sendo $n_{a} \mathrm{o}$ número de alelos no loco) são utilizados (Ritland \& Jain 1981). A taxa de cruzamento multilocos foi estimada a partir de sete locos polimórficos, sendo que em cada loco, pelo menos dois alelos eram comuns (freqüência $>0,1$ ). Portanto, o conjunto multilocos era adequado para estimar a taxa de cruzamento. Em concordância, o erro padrão associado à estimativa da taxa de cruzamento multilocos foi baixo $(0,040)$, podendo-se considerar a estimativa obtida, robusta. Desta forma, se existe auto-incompatibilidade na espécie, ela deve ser fraca ou restrita a certos períodos de florescimento das plantas, ou ainda, estar associada a certos genótipos na população. Em Eucalyptus globulos L. ocorrem tanto plantas auto-incompatíveis como autocompatíveis (Patterson et al. 2001). Isto também pode ser o caso de $C$. speciosa e explicaria as diferenças nos resultados aqui obtidos em relação aos observados por Gibbs \& Bianchi (1993).

A taxa de cruzamento multilocos estimada em C. speciosa $(0,816)$ é comparável a encontrada em 
outras três espécies arbóreas tropicais da Família Bombacaceae. Em Cavanillesia platanifolia (H. \& B.) H.B.K. (Murawski et al. 1990) a taxa de cruzamento multilocos $\left(\hat{t}_{m}\right)$ variou de 0,569 , em 1987, a 0,347 , em 1988, uma das mais baixas taxas detectadas em espécies arbóreas. Em Quararibea asterolepis Pitt. (Murawski et al. 1990) a taxa de cruzamento foi igual à unidade $(1,008)$, porém, na estimativa da taxa de cruzamento individual por árvore materna foram observados valores inferiores à unidade, mostrando que apesar da alta taxa de cruzamento populacional, seu sistema de reprodução também era misto. Em Ceiba pentandra (L.) Gaertn. (Murawski \& Hamrick 1992) a taxa $\hat{t}_{m}$ variou de 0,898 a 0,924 , também sugerindo reprodução por sistema misto $(t<0,95)$. A baixa taxa de cruzamento observada em $C$. platanifolia foi atribuída à ausência de mecanismos de auto-incompatibilidade e à baixa densidade populacional (Murawski et al. 1990). Estes fatores, de alguma forma, podem também ter contribuído para os resultados observados em C. speciosa. A espécie ocorre na população avaliada, em baixa densidade (menos de um indivíduo por hectare), o que poderia ter favorecido a ocorrência de autofecundação.

O teste de Equilíbrio de Hardy-Weinberg e a estimativa da divergência genética $\left(\hat{F}_{S T}\right)$ entre as freqüências alélicas de adultos vs. progênies, adultos vs. pólen e pólen vs óvulos, evidenciaram desvios de cruzamentos aleatórios. Desvios do EHW podem ser causados pelo sistema misto de reprodução ou fatores evolucionários como deriva genética, seleção e mutação. Considerando a natureza supostamente neutra dos marcadores isoenzimáticos e que a taxa média de substituição de códons detectáveis por isoenzimas é, na maioria das vezes, baixa, $10^{-7}$ (Nei 1975), logo, teria pouca força para causar as alterações observadas, os desvios podem ser atribuídos ao sistema de reprodução e/ou à deriva genética. O sistema de reprodução foi aparentemente o maior responsável pelos desvios, visto que as maiores alterações foram observadas nas freqüências genotípicas, como evidenciado pelo alto índice de fixação nas progênies e não pelas alterações nas freqüências alélicas entre gerações. $O$ sistema de reprodução não altera as freqüências alélicas, apenas as freqüências genotípicas. Já, a deriva genética, pode alterar tanto as freqüências alélicas como as genotípicas por erros de amostragem. As freqüências alélicas entre adultos e progênies foram estatisticamente diferentes em dois locos (Pgi-1,
Prx-1), apesar da baixa divergência genética detectada nesses locos (máximo 1,1\%). Neste caso, a significância estatística parece estar mais associada ao tamanho amostral e ao método de cálculo do teste de qui-quadrado para o estimador $F_{S T}$ (Workman \& Niswander 1970), do que devido a uma diferença biológica. Uma divergência genética de $1 \%$ pode ser considerada de pouco efeito, do ponto de vista biológico, portanto, a deriva genética deve ter tido pouco efeito nos desvios do EHW observados nas progênies. Ainda, analisando a expressão de qui-quadrado, $\chi^{2}=2 n F_{S T}(k-1)$, usada para testar a significância estatística de $F_{S T}$, verifica-se que esta é sensível ao tamanho amostral, podendo, para locos com mesmos valores de $F_{S T}$ e mesmo número de alelos, apresentar resultados diferentes. Se o estimador $F_{S T}$ for baixo, somente grandes tamanhos amostrais detectarão significância estatística, e se o estimador $F_{S T}$ for alto, pequenos tamanhos amostrais poderão ser capazes de detectar significância estatística. O teste de equilíbrio de endogamia em locos neutros permite separar os efeitos do sistema de reprodução dos efeitos da deriva genética, nos desvios do EHW. Entretanto, não foi possível testar a hipótese de equilíbrio de endogamia, porque os locos que apresentaram desvios do modelo de EHW foram justamente os locos que continham apenas dois alelos, e são necessários pelo menos três alelos no loco para ter-se graus de liberdade suficientes para o teste. No teste de equilíbrio de endogamia, um grau de liberdade é perdido em função da estimativa de $F$, um devido ao número de classes genotípicas e um outro devido ao número de alelos no loco, logo, em locos com apenas dois alelos não sobram graus de liberdade para a realização do teste, GL = [(3 classes- 1$)-(2$ alelos -1$)-1]=0$.

O sistema misto de reprodução tem sido, geralmente, conceituado como a mistura de cruzamentos aleatórios e autofecundações. No entanto, esta conceituação desconsidera a ocorrência de cruzamentos biparentais, uma outra forma de reprodução que também pode causar desvios de cruzamentos aleatórios, mesmo em espécies obrigatoriamente de cruzamento (espécies dióicas). Os cruzamentos biparentais podem ser medidos pela correlação de paternidade ( $r_{p}$; Ritland 1989). A correlação $r_{p}$ mede a proporção de indivíduos de cruzamentos que foram gerados por cruzamentos biparentais (irmãos completos (Ritland 1989)). A estimativa da correlação $r_{p}$ em $C$. speciosa foi alta 
$(0,875)$, sugerindo que grande parte das progênies de cruzamentos foram geradas por cruzamentos biparentais (tabela 3), ou seja, foram geradas pelo mesmo parental materno e paterno. Poucos trabalhos realizados com espécies arbóreas tropicais reportam estimativas das correlações $r_{p}$. Em Esenbeckia leiocarpa Engl. (Seoane et al. 2001) as estimativas das correlações de paternidade variaram entre populações de 0,749 a 0,986 , umas das mais altas detectadas em espécies arbóreas tropicais. Em Cariniana legalis (Mart.) O. Ktze. (Sebbenn et al. 2000) as correlações de paternidade variaram entre populações de 0,212 a 0,324. Em Tabebuia cassinoides (Lam.) A.P. De Candolle (Sebbenn et al. 2001) foi detectada maior correlação de paternidade em uma população natural $(0,547)$, em comparação a uma população manejada $(0,295)$. A causa dos cruzamentos biparentais pode ser atribuída ao comportamento dos polinizadores visitando de forma sistemática árvores próximas, poliandria ou depósito de múltiplos grãos de pólen de um simples polinizador e pequeno número de vizinhos próximos (Sun \& Ritland 1998) ou assincronismo no florescimento das árvores. Possivelmente, vários destes fatores tenham contribuído para a alta taxa de cruzamento biparental observadas em C. speciosa. A baixa densidade populacional na área estudada pode ser uma das causas prováveis dos cruzamentos biparentais (um indivíduo reprodutivo a cada dois hectares). Murawski \& Hamrick (1991) observaram que, em populações de espécies arbóreas de baixa densidade, o pólen dos cruzamentos era menos diversificado do que em espécies de alta densidade. Outra causa pode ser o comportamento e/ou a densidade de polinizadores. Variações ambientais podem afetar o comportamento e/ou a densidade das populações de polinizadores, levando a alterações na taxa de cruzamento das espécies em diferentes populações (Murawski 1995).

Uma alta taxa de correlação de paternidade implica que grande parte das progênies são aparentadas no grau de irmãos completos. A taxa de cruzamento multilocos $\left(\hat{t}_{m}\right)$ obtida para C. speciosa, combinada com a correlação de paternidade $\left(\hat{r}_{p}\right)$, permite afirmar que aproximadamente $18,4 \%$ das progênies analisadas foram originadas por autofecundação $\left(1-\hat{t}_{m}\right)$ sendo sua relação de parentesco de irmãos de autofecundação, $71,4 \%$ por cruzamentos biparentais $\left(\hat{t}_{m} \hat{r}_{p}\right)$, sendo aparentadas no grau de irmãos completos, e somente $10,2 \%$ por cruzamentos aleatórios $\left[\hat{t}_{m}\left(1-\hat{r}_{p}\right)\right]$, sendo aparentadas no grau de meio-irmãos. Isto mostra que as progênies de C. speciosa são na maioria irmãos completos. Em concordância, o valor estimado para o coeficiente de coancestralidade dentro de progênies $\left(\hat{\theta}_{F}=0,253\right)$ atingiu valor próximo ao esperado por progênies de irmãos completos $(0,25)$. Entretanto, tendo em vista que foram detectados $5,6 \%$ de cruzamentos entre indivíduos aparentados, parte das progênies geradas por cruzamentos aleatórios e biparentais devem ser aparentadas em grau superior aos de meio-irmãos e irmãos completos.

A estimativa do índice de fixação mostrou maior endogamia nas progênies $\left(F_{p}=0,259\right)$, em comparação às árvores maternas $\left(F_{m}=0,073\right)$ e à população adulta $(F=0,142)$. Assumindo o equilíbrio de endogamia e estimando o coeficiente de endogamia em equilíbrio, $F_{e}=(1-t) /(1+t)$ (Allard et al. 1968), conclui-se que a taxa detectada de autofecundação, 0,184 $\left(s=1-\hat{t}_{m}\right)$, poderia ter gerado no máximo 0,101 de endogamia. Assim, a endogamia estimada nas progênies foi aproximadamente 2,5 vezes maior do que a esperada em equilíbrio. $\mathrm{O}$ excesso de endogamia pode ter sido causada pelo acúmulo de endogamia entre geração (autofecundação de indivíduo endogâmicos) e pelos cruzamentos entre parentes. Os índices de fixação estimados nas árvores maternas $\left(F_{m}=0,073\right)$ e na população adulta ( $F=0,142)$, apesar de não serem estatisticamente diferentes de zero, sugerem a possibilidade da população ser endogâmica, o que, aliado à taxa estimada de cruzamento entre parentes $(5,6 \%)$ poderiam explicar, em parte, a endogamia observada. Outra causa poderia ser o grau de parentesco dentro da população. Admitindo o equilíbrio de endogamia, o coeficiente de endogamia nas progênies corresponde ao coeficiente de parentesco na geração parental $(\theta=F)$. Desta forma, o valor do coeficiente de parentesco na população adulta seria de 0,259 , o que corresponde ao coeficiente de parentesco entre irmãos completos. Os cruzamentos entre irmãos completos poderiam ter contribuído para a endogamia detectada nas progênies.

A endogamia pode ter efeitos negativos sobre a capacidade adaptativa e reprodutiva de populações pequenas $(N<100)$. Em espécies arbóreas estes efeitos são incrementados pela sobreposição de gerações. Em populações pequenas, na ausência de seleção contra homozigotos, a taxa de endogamia e parentesco pode crescer rapidamente, levando à depressão endogâmica. Suas principais características são a perda de vigor, fertilidade, capacidade de adaptação e reprodução. Em espécies arbóreas os efeitos negativos da depressão 
endogâmica são bem documentados (Libby et al. 1981, Matheson et al. 1995, Wu et al. 1998). Em contrapartida, em C. speciosa existem fortes evidências de seleção contra homozigotos entre a fase de plântula e a fase adulta. $\mathrm{O}$ índice de fixação estimado nas progênies $(0,259)$ foi maior do que o estimado nos adultos $(0,142)$ e nas árvores maternas $(0,073)$. Seleção contra homozigotos também tem sido evidenciada em vários outros estudos (Murawski et al. 1990, Murawski \& Hamrick 1992, Murawski 1995, Sebbenn et al. 2001). A seleção poderia eliminar parte da endogamia gerada pelas autofecundações e cruzamentos entre parentes, mantendo a variabilidade genética das populações.

Progênies de polinização aberta são, na maioria das vezes, assumidas por serem originadas de cruzamentos aleatórios, o que resultaria em progênies de meio-irmãos. Os resultados observados para C. speciosa discordam desta hipótese, mostrando que a maior parte das progênies são irmãos completos, sendo o restante irmãos de autofecundação e meio-irmãos. Este resultado tem implicações importantes na estimativa e parâmetros genéticos em caracteres quantitativos. A estimativa da variância genética aditiva e parâmetros dependentes desta, como herdabilidades e ganhos esperados não seleção, serão superestimados, caso as progênies sejam assumidas como meio-irmãos (Namkoong 1966). Se progênies de polinização livre são coletadas de populações sem endogamia e parentesco, a correlação genética entre plantas dentro de progênies $\left(r_{x y}\right)$ estima 1/4 da variância genética aditiva (Namkoong 1966). Se ocorrerem autofecundações e/ou cruzamentos biparentais, a correlação $r_{x y}$, não é mais $1 / 4$. Substituindo-se os parâmetros estimados em C. speciosa (tabela 3), no estimador $r_{x y}$ proposto por Ritland (1989), para espécies de reprodução mista, $r_{x y}=1 / 4(1+F)\left[4 s+\left(t^{2}+s t r_{s}\right)\left(1+r_{p}\right)\right]$, chega-se ao valor 0,507, que é aproximadamente o dobro do esperado em progênies de meio-irmãos $(0,25)$. Ainda, como o coeficiente de correlação é duas vezes o coeficiente de coancestria entre plantas dentro de progênies $\left(r_{x y}=2 \theta_{F}\right)$, tem-se que $\theta_{F}=r_{x y} / 2$, resultando em 0,253 , valor idêntico ao coeficiente de coancestria estimado pela análise da variância das freqüências alélicas (tabela 3 ).

O sistema misto tem também implicações na estimativa do tamanho efetivo de variância $\left(N_{e}\right)$, o qual mede a representatividade genética de uma população em função de alterações nas freqüências alélicas entre gerações por efeitos da deriva genética.
Por exemplo, se as árvores na população de C. speciosa não fossem aparentadas e endogâmicas e infinitas sementes fossem coletadas nas 28 progênies amostradas, o tamanho efetivo aproximar-se-ia de $4 m$, sendo $m$ o número de progênies, logo, $N_{e}=112$. Por outro lado, para as mesmas condições anteriores (coleta de infinitas sementes em população não endogâmica e sem parentesco), usando-se a expressão de tamanho efetivo de variância derivada por Cockerham (1969) para espécies hermafroditas de sistema misto, $N_{e}=0,5 m /\left[\theta_{F}(n-1 / n)+\left(1+F_{p}\right) / 2 n\right]$, em que, $\theta_{F}$ é o coeficiente médio de coancestria dentro de progênies, $n$ é o tamanho total da amostra e $F_{p}$ é o índice de fixação nas progênies, e substituindo-se seus respectivos valores estimados na tabela 3 , chegase ao tamanho efetivo de 53,3. Conclui-se assim, que o tamanho efetivo estimado, considerando os parâmetros do sistema de reprodução, é aproximadamente $100 \%$ menor do que o esperado por cruzamentos aleatórios (112). Estes resultados, portanto, mostram que é fundamental o conhecimento prévio do sistema de reprodução das espécies, em programas de melhoramento e conservação genética ex situ.

Agradecimentos - Os autores agradecem ao Instituto Florestal pela permissão para a amostragem na Estação Ecológica de Bauru-SP e à Fapesp pelo suporte financeiro e concessão da bolsa de mestrado para o primeiro autor.

\section{Referências bibliográficas}

ALLARD, R.W., JAIN, S.K. \& WORKMAN, P.L. 1968. The genetics of inbreeding populations. Advance in Genetics 14:55-131.

ALFENAS, A.C. 1998. Eletroforese de isoenzimas e proteínas afins: Fundamentos e aplicações em plantas e microrganismos. Editora UFV, Viçosa.

BAWA, K.S. \& O’MALLEY, D.M. 1985. Estudios geneticos y sistemas de cruzamiento en algunas especies arboreas de bosques tropicales. Revista de Biologia Tropical 35:177-188.

CARVALHO, P.E.R. 1994. Espécies florestais brasileiras: Recomendações silviculturais, potencialidades e uso de madeira. Embrapa-CNPF, Brasília.

CASTILlO, C.A.R. 1986. Dispersão anemocórica das sementes de paineira (Chorisia speciosa St. Hil.) na região de Bauru, Estado de São Paulo. Dissertação de mestrado, Escola Superior de Agricultura Luiz de Queiroz, Piracicaba. 
CLAYTON, J. \& TRETIAK, D. 1972. Amine-citrate buffers for $\mathrm{pH}$ control in starch gel electrophoresis. Journal Fisheries Research Board Canadian 29:1169-1172.

COCKERHAM, C.C. 1969. Variance of gene frequencies. Evolution 23:72-84.

GIBBS, P.E. \& BIANCHI, M. 1993. Post-pollination events in species of Chorisia speciosa (Bombacaeae) and Tabebuia (Bignoniaceae) with late-actioning self-incompatibility. Botanica Acta 106:64-71.

HAMRICK, J.L. \& LOVELESS, M.D. 1986. The influence of seed dispersal mechanisms on the genetic structure of plant populations. In Frugivores and seed dispersal (A. Estrada \& T.H. Fleming, eds.). Junk Publishers, New York, p.17-59.

HAMRICK, J.L. 1983. The distribution of genetic variation within and among natural plant population. In Genetics and conservation (C.M. Schone-Wald-Cox, S.H. Chambers, B. MacByde \& L. Thomas, eds.). Benjamin Cummings Publishing Company, Menlo Park, p.335-348.

HAMRICK, J.L. \& GODT, M.J.W. 1989. Allozyme diversity in plant species. In Plant population genetics, breeding and genetic resources (A.H.D. Brown, M.T. Clegg, A.L. Kahler \& B.S. Weir, eds). Sinauer, Sunderland, p.43-63.

KEPHART, S.R. 1990. Starch gel eletrophoresis of plant isozymes: a comparative analysis of techniques. American Journal of Botany 77:693-712.

LEWIS, P.O. \& ZAYKIN, D. 1999. Genetic date analysis: computer program for the analysis of allelic date. Versão 1.0 para Windows 3.1. Free program distributed by the authors over the Internet from the Genetic Date Analysis home page at $<$ http//alleyn.eeb.uconn.edu/gda/ $>$.

LIBBY, W.J., McCUTCHAN, B.G. \& MILLAR, C.I. 1981. Inbreeding depression in selfs of Redwood. Silvae Genetica 30:15-25.

MATHESON, A.C., WHITE, T.L. \& POWEL, G.R. 1995. Effects of inbreeding on growth, stem form and rust resistence in Pinus elliottii. Silvae Genetica 44:37-46.

MORELLATO, L.P.C. 1991. Estudo da fenologia de árvores e lianas de uma floresta semidecídua no sudoeste do Brasil. Tese de doutorado, Universidade Estadual de Campinas, Campinas.

MURAWSKI, D.A., HAMRICK, J.L., HUBBELL, S.P. \& FOSTER, R.B. 1990. Mating system of two Bombacaceous trees of a neotropical moist forest. Oecologia 82:501-506.

MURAWSKI, D.A. \& HAMRICK, J.L. 1991. The effect of the density of flowering individuals on the mating systems of nine tropical tree species. Heredity 67:167-174.

MURAWSKI, D.A. \& HAMRICK, J.L. 1992. Mating system and phenology of Ceiba pentandra (Bombacaceae) in central Panama. Journal of Heredity 83:401-404.
MURAWSKI, D.A. 1995. Reproductive biology and genetics of tropical trees from a canopy perspective. In Forest canopies (M.D. Lowman \& N.M. Nadkarmi, eds.). Academic Press, New York, p.457-493.

NAMKOONG, G. 1966. Imbreed effects on estimation of genetic additive variance. Forest Science 12:8-13.

NEI, M. 1975. Molecular populations genetics and evolution. North-Holland Publishing Co., Amsterdam.

NEI, M. 1977. F-Statistic and analysis of gene diversity in subdividid populations. Annual Human Genetics 41:225-33.

PATTERSON, B., VAILLANCOURT, R.E. \& POTTS, B.M. 2001. Eucalypts seed collectors: beware of sompling seedlots from low in the canopy! Australian Forestry 64:139-142.

RITLAND, K. \& JAIN, S. 1981. A model for the estimation of outcrossing rate and gene frequencies using independent loci. Heredity 47:35-52.

RITLAND, K. 1989. Correlated matings in the partial selfer Mimulus guttatus. Evolution 43:848-859.

RITLAND, K. 1997. Multilocus mating system program MLTR. Version 1.1. University of British Columbia, Canadá. Free program distributed by the authors over the Internet from the Kermit Ritland home page at $<$ http// genetics.forestry.ubc.ca/ritland/programs.html $>$.

SEOANE, C.E.C., SEBBENN, A.M. \& KAGEYAMA, P.Y. 2001. Sistema reprodutivo em populações de Esenbeckia leiocarpa. Revista do Instituto Florestal 13:21-28.

SEBBENN, A.M., KAGEYAMA, P.Y., SIQUEIRA, A.C.M.F., \& ZANATTO, A.C.E. 2000. Taxa de cruzamento em populações de Cariniana legalis (Mart.) O. Ktze.: implicações para a conservação e o melhoramento genético. Scientia Forestalis 58:25-40.

SEBBENN, A.M., SEOANE, C.E., KAGEYAMA, P.Y. \& LACERDA, M.C.B. 2001. Estrutura genética em populações de Tabebuia cassinoides: implicações para o manejo florestal e a conservação genética. Revista do Instituto Florestal 13:99-113.

SUN, M. \& RITLAND, K. 1998. Mating system of yellow starthistle (Centaurea solstitialis), a successful colonizer in North America. Heredity 80:225-232.

WORKMAN, P. \& NISWANDER, J.L. 1970. Population studies on southwestern indian tribes. II. Local genetic differentiation in the Papago. American Journal Human Genetic 22:24-49.

WU, H.X., MATHESON, A.C. \& SPENCER, D. 1998. Inbreeding in Pinus radiata. 1. The effect of inbreeding on growth, survival and variance. Theorical and Applied Genetic 97:1256-1268. 